\title{
AN ACTION OF THE SYMPLECTIC MODULAR GROUP
}

\section{LOUIS SOLOMON*}

To the memory of TADASI NAKAYAMA

1. Let $V$ be a free $\mathbf{Z}$-module of $\operatorname{rank} 2 n$. Let $G=\mathbf{S p}(2 n, \mathbf{Z})$ be the symplectic modular group and let $\Phi$ be the non-singular alternating bilinear form on $V$ left invariant by $G$. Let $p \in \mathbf{Z}$ be a prime and let $X$ be the set of all endomorphisms $\xi$ of $V$ such that

$$
\Phi(\xi x, \xi y)=p \Phi(x, y)
$$

for all $x, y \in V$. In the theory of transformation of theta functions [3] one encounters the natural action of $G$ on $X$ by left multiplication. The number of $G$ orbits is known to be finite and the point of this note is a proof of the following

Theorem. The number of orbits of $X$ under $G$ is $\prod_{i=1}^{n}\left(1+p^{i}\right)$

The case $n=2$ is due to Hermite [2] and the case $n=3$ to Weber [4] who compute explicit sets of representatives for the orbits in these cases. The idea in the present argument is to reduce the problem to a question about the finite symplectic group $\operatorname{Sp}\left(2 n, \mathbf{F}_{p}\right)$. In the new situation Witt's theorem is available for counting purposes. The number $\prod_{i=1}^{n}\left(1+p^{i}\right)$ is the number of maximal totally isotropic subspaces of a $2 n$ dimensional symplectic space over $\mathbf{F}_{p}$.

2. Let $V$ be a free $\mathbf{Z}$-module of rank $2 n$. Let $\Phi: V \times V \rightarrow \mathbf{Z}$ be a nonsingular alternating bilinear form on $V$. We assume that $V$ has a basis $v_{1}$, $\ldots, v_{2 n}$ such that the matrix of $\mathscr{D}\left(v_{i}, v_{j}\right)$ is

$$
J=\left(\begin{array}{rr}
0 & I \\
-I & 0
\end{array}\right)
$$

where $I$ is the identity matrix of degree $n$. We call $v_{1}, \ldots, v_{2 n}$ a symplectic basis for $V$. The symplectic modular group $\mathbf{S p}(2 n, \mathbf{Z})$ consists of all automor-

Received March 22, 1965.

* This work was supported in part by the National Science Foundation under grant GP 1452 . 
phisms $\tau$ of $V$ such that $\emptyset(\tau x, \tau y)=\emptyset(x, y)$ for all $x, y \in V$. We let $\mathbf{F}=\mathbf{Z} / p \mathbf{Z}$ denote the field of $p$ elements and set $E=V / p V$. We view $E$ as vector space over $\mathbf{F}$. The form $\Phi$ defines, by reduction $\bmod p$, a non-singular alternating bilinear form $\Psi: E \times E \rightarrow \mathbf{F}$. Similarly, an endomorphism of $\operatorname{Homz}(V, V)$ defines an endomorphism of $\operatorname{Hom}(E, E)$. In this way we get a homomorphism of $\mathbf{S p}(2 \boldsymbol{n}, \mathbf{Z})$ into the group $\mathbf{S p}(2 \boldsymbol{n}, \mathbf{F})$ of all non-singular $\mathbf{F}$-linear transformations of $E$ which preserve the form $\Psi$. A transvection

$$
\tau: v \rightarrow v+a \Phi(v, w) w \quad w \in V, \boldsymbol{a} \in \mathbf{Z}
$$

of $\mathbf{S p}(2 n, \mathbf{Z})$ maps into a transvection of $\mathbf{S p}(2 n, \mathbf{F})$. Since every transvection in $\mathbf{S p}(2 n, \mathbf{F})$ may be obtained in this way by reduction $\bmod p$, and since the transvections generate $\mathbf{S p}(2 \boldsymbol{n}, \mathbf{F})$ we see that the map $\mathbf{S p}(2 n, \mathbf{Z}) \rightarrow \mathbf{S p}(2 n, \mathbf{F})$ is an epimorphism. We use $x \rightarrow x^{*}$ as a notation for each of the reductions

$$
\mathbf{Z} \rightarrow \mathbf{F}, \quad V \rightarrow E, \quad \mathbf{S p}(2 n, \mathbf{Z}) \rightarrow \mathbf{S p}(2 n, \mathbf{F})
$$

modulo $p$. We use those facts about symplectic spaces over a field which center around Witt's theorem. These facts are proved in [1].

Lemma 1. Let $e_{1}, \ldots, e_{2 n}$ be a symplectic basis for $E$. Then there exists a symplectic basis $w_{1}, \ldots, w_{\text {in }}$ for $V$ such that $w_{i}^{*}=e_{i}$.

Proof. Let $v_{1}, \ldots, v_{2 n}$ be a symplectic basis for $V$. Then $v_{1}^{*}, \ldots, v_{2 n}^{*}$ is a symplectic basis for $E$. Define an F-linear transformation $\beta$ of $E$ by $\beta v_{i}^{*}$ $=e_{i}$. Then $\beta \in \mathbf{S p}(2 n, \mathbf{F})$ and, since $\operatorname{Sp}(2 n, \mathbf{Z})$ maps onto $\operatorname{Sp}(2 n, \mathbf{F})$ we may choose $\alpha \in \mathbf{S p}(2 n, \mathbf{Z})$ with $\alpha^{*}=\beta$. Set $w_{i}=\alpha v_{i}$. Then $w_{1}, \ldots, w_{2 n}$ is a symplectic basis for $V$ and $w_{1}^{*}=\alpha^{*} v_{1}^{*}=e_{i}$.

Lemma 2. Let $\xi \in X$. Then Ker $\xi^{*}$ and Im $\xi^{*}$ are maximal totally isotropic subspaces of $E$.

Proof. If $a, b \in \operatorname{Ker} \xi^{*}$ choose $x, y \in V$ with $x^{*}=a, y^{*}=b$. Then $\xi x, \xi y \in p V$ so $\xi x=p x^{\prime}, \xi y=p y^{\prime}$ for some $x^{\prime}, y^{\prime} \in V$. Then

$$
p \Phi(x, y)=\Phi(\xi x, \xi y)=p^{2} \Phi\left(x^{\prime}, y^{\prime}\right) \in p^{2} \mathbf{Z}
$$

so $\mathscr{D}(x, y) \in p \mathrm{Z}$ and $\Psi(a, b)=0$. Thus $\operatorname{Ker} \xi^{*}$ is totally isotropic. Similarly, if $a, b \in \operatorname{Im} \xi^{*}$ write $a=\xi^{*} x^{*}, b=\xi^{*} y^{*}$ for some $x, y \in V$ and then

$$
\Psi(a, b)=\emptyset(\xi x, \xi y)^{*}=p^{*} \Phi(x, y)^{*}=0
$$


so that $\operatorname{Im} \xi^{*}$ is totally isotropic. We must prove that $\operatorname{dim} \operatorname{Ker} \xi^{*}=n=\operatorname{dim}$ $\operatorname{Im} \xi^{*}$.

Let $T$ be the matrix for $\xi$ in the symplectic basis $v_{1}, \ldots, v_{2 n}$. Since $\Phi(\xi x, \xi y)=p \mathscr{D}(x, y)$ we have $T J T^{t}=p J$ where $T^{t}$ denotes the transpose of $T$. Thus $(\operatorname{det} T)^{2}=p^{2 n}$ so $|\operatorname{det} \xi|=p^{n}$. Imbed $V$ in the vector space $V \otimes \mathbf{Q}$ over the rational field $\mathbf{Q}$. Then $\emptyset$ extends to a form, denoted again $\emptyset$, on $V \otimes \mathbf{Q}$ and $\xi$ defines a linear transformation, denoted again $\xi$, of $V \otimes \mathbb{Q}$. Then $\emptyset(\xi x, \xi y)$ $=p \Phi(x, y)$ for all $x, y \in V \otimes \mathbf{Q}$. Since det $\xi \neq 0, \xi$ is invertible, and for any $x, v \in V$ we have

$$
\Phi\left(\xi^{-1} p x, v\right)=\emptyset\left(\xi^{-1} p x, \xi^{-1} \xi v\right)=p^{-1} \emptyset(p x, \xi v)=\emptyset(x, \xi v) \in \mathbf{Z}
$$

Now letting $v$ range over a symplectic basis for $V$ we see that $\xi^{-1} p x \in V$. Thus $\xi^{-1} p V \subseteq V$, so $p V \subseteq \xi V$. Let $d_{1}, \ldots, d_{2 n} \in \mathbf{Z}$ be the elementary divisors of $\xi$ viewed as endomorphism of $V$, where we choose the $d_{i}$ non-negative and such that $d_{i+1}$ divides $d_{i}$. Choose $\mathbf{Z}$-bases $x_{1}, \ldots, x_{2 n}$ and $y_{1}, \ldots, y_{2 n}$ for $V$ so that $\xi x_{i}=d_{i} y_{i}$. Since $p V \subseteq \xi V$ we must have $p y_{i} \in \mathbf{Z} d_{i} y_{i}$ so each $d_{i}$ divides p. But $d_{1}, \ldots, d_{2 n}=|\operatorname{det} \xi|=p^{n}$ so we have $d_{1}=\cdots=d_{n}=p$ and $d_{n+1}=\cdot$ $\cdots=d_{2 n}=1$. Thus the elementary divisors of $\xi^{*}$ are $d_{1}^{*}=\cdots=d_{n}^{*}=0$ and $\boldsymbol{d}_{n+1}^{*}=\cdots=d_{2 n}^{*}=1$. Thus $\xi^{*}$ has rank $n$ and hence $\operatorname{dim} \operatorname{Ker} \xi^{*}=n=\operatorname{dim} \operatorname{Im} \xi^{*}$. This proves the lemma.

Lemma 3. Suppose $\xi, \eta \in X$. If Ker $\xi^{*}=\operatorname{Ker} \eta^{*}$ then $\xi$ and $\eta$ are in the same orbit under $G$.

Proof. Lemma 2 tells us that $D=\operatorname{Ker} \xi^{*}$ is a maximal totally isotropic subspace of $E$. The theorem on Witt decomposition of symplectic spaces over a field asserts the existence of a maximal totally isotropic subspace $D^{\prime}$ of $E$ such that $E=D+D^{\prime}$, direct sum. Furthermore there exist bases $e_{1}, \ldots, e_{n}$ for $D$ and $e_{n+1}, \ldots, e_{2 n}$ for $D^{\prime}$ such that $e_{1}, \ldots, e_{2 n}$ is a symplectic basis for $E$. Lemma 1 shows the existence of a symplectic basis $w_{1}, \ldots, w_{2 n}$ for $V$ such that $w_{i}^{*}=e_{i}$. Define $\theta \in X$ by

$$
\begin{array}{ll}
\theta w_{i}=p w_{i} & i=1, \ldots, n \\
\theta w_{i}=w_{i} & i=n+1, \ldots, 2 n
\end{array}
$$

Then $\operatorname{Ker} \theta^{*}=D$. Since $\operatorname{Im} \xi^{*}=\xi^{*} D^{\prime}$ and $\operatorname{Im} \theta^{*}=\theta^{*} D^{\prime}$ we see from Lemma 2 that $\xi^{*} D^{\prime}$ and $\theta^{*} D^{\prime}$ are totally isotropic subspaces of $E$ of the same dimension 
n. Define a non singular F-linear transformation $\beta: \xi^{*} D^{\prime} \rightarrow \theta^{*} D^{\prime}$ by $\beta \xi^{*} e_{i}=\theta^{*} e_{i}$ for $i=n+1, \ldots, 2 n$. Since $\xi^{*} D^{\prime}$ and $\theta^{*} D^{\prime}$ are totally isotropic, $\beta$ is an isometry, and, by Witts theorem, may be extended to an element, denoted again $\beta$, of $\operatorname{Sp}(2 n, \mathbf{F})$. Since both $\xi^{*}$ and $\theta^{*}$ annihilate $D$ we have $\beta \xi^{*} e_{i}=\theta^{*} e_{i}$ for all $i=1, \ldots, 2 n$ so $\beta \xi^{*}=\theta^{*}$. Choose $\alpha \in \mathbf{S p}(2 n, \mathbf{Z})$ with $\alpha^{*}=\beta$. Then $(\alpha \xi)^{*}$ $=\alpha^{*} \xi^{*}=\theta^{*}$. In particular $\alpha \xi w_{i} \in p V$ for $i=1, \ldots, n$. Define $\sigma \in \operatorname{Hom}_{\mathbf{Z}}(V, V)$ by

$$
\begin{array}{rlrl}
\sigma w_{i} & =\frac{1}{p} \alpha \xi w_{i} & i=1, \ldots, n \\
\sigma w_{i}=\alpha \xi w_{i} & i=n+1, \ldots, 2 n
\end{array}
$$

Then $\alpha \xi=\sigma \theta$ so

$$
\emptyset(\sigma \theta x, \sigma \theta y)=\emptyset(\alpha \xi x, \alpha \xi y)=p \emptyset(x, y)=\emptyset(\theta x, \theta y)
$$

for all $x, y \in V$. Since $\theta V$ has finite index in $V$ the bilinearity of $\Phi$ implies $\Phi(\sigma x, \sigma y)=\Phi(x, y)$ for all $x, y \in V$. Now, as in the proof of Lemma 1 , we conclude $\operatorname{det} \sigma=1$ so that $\sigma \in G$. Thus we have shown the existence of $\alpha, \sigma \in G$ with $\alpha \xi=\sigma \theta$. Similarly there exist $\beta, \tau \in G$ with $\beta \eta=\tau \theta$. Then $\eta=\beta^{-1} \tau \sigma^{-1} \alpha \xi$ so that $\xi, \eta$ lie in the same orbit under $G$.

Proposition. The map $\xi \rightarrow$ Ker $\xi^{*}$ induces a one to one correspondence between orbits of $X$ under $G$ and maximal totally isotropic subspaces of $E$.

Proof. If $\xi, \eta \in X$ lie in the same orbit, say $\xi=\tau \eta$ with $\tau \in \mathbf{S p}(2 n, \mathbf{Z})$. Then $\xi^{*}=\tau^{*} \eta^{*}$. Since $\tau^{*} \in \mathbf{S p}(2 n, \mathbf{F})$ is non-singular we have $\operatorname{Ker} \xi^{*}=\operatorname{Ker} \eta^{*}$. Thus $\xi \rightarrow \operatorname{Ker} \xi^{*}$ induces a map of orbits into the set of maximal totally isotropic subspaces of $E$. By Lemma 3 the map is one to one. To see that every maximal totally isotropic subspace $D$ occurs as a kernel of some $\xi^{*}$, construct a Witt decomposition $E=D+D^{\prime}$ as in the proof of Lemma 3 , and note that the element $\theta \in X$ satisfies $\operatorname{Ker} \theta^{*}=D$. This completes the proof.

3. We have thus reduced the problem to computing the number $t$ of maximal totally isotropic subspaces of a $2 n$ dimensional symplectic space over F. The finite group $\mathbf{S p}(2 \boldsymbol{n}, \mathbf{F})$ acts as a permutation group on the set of maximal totally isotropic subspaces of $E$. By Witt's theorem this permutation group is transitive, hence

$$
t=|G: H|
$$


where $H$ is the group of all $\gamma \in \mathbf{S p}(2 n, \mathbf{F})$ which leave globally invariant a given maximal totally isotropic subspace $D$. The restriction map $\gamma \rightarrow \gamma \mid D$ defines a homomorphism of $H$ into the full linear group $\mathbf{G L}(D)=\mathbf{G L}(n, \mathbf{F})$. By Witt's theorem this is an epimorphism. The kernel $K$ consists of those elements of $\mathbf{S p}(2 n, \mathbf{F})$ which fix $D$. It is known, and it is easy to compute directly, that $K$ is isomorphic to the additive group of $n \times n$ symmetric matrices over $\mathbf{F}$ so that $K$ has order $|K|=p^{n(n+1) / 2}$. Thus

$$
t=|\mathbf{S p}(2 n, \mathbf{F}) \| \mathbf{G L}(n, \mathbf{F})|^{-1} p^{-n(n+1 / / 2}
$$

If we insert the known formulas

$$
\begin{aligned}
& |\mathbf{S p}(2 n, \mathbf{F})|=p^{n^{2}} \prod_{i=1}^{n}\left(p^{2 i}-1\right) \\
& |\mathbf{G L}(\boldsymbol{n}, \mathbf{F})|=p^{n(n-1) / 2} \prod_{i=1}^{n}\left(p^{i}-1\right)
\end{aligned}
$$

we find

$$
t=\prod_{i=1}^{n}\left(1+p^{i}\right)
$$

This proves the theorem.

\section{REFERENCES}

[1] E. Artin, Geometric Algebra, Interscience, Ncw York (1957).

[2] C. Hermite, Sur la théorie de la transformation des fonctions abéliennes, Comptes Rendus 40 (1855), 249-254.

[ 3 ] A. Krazer, Lehrbuch der Thetafunktionen, Teubner, Leipzig (1903).

[4] H. Weber, Über die Transformationstheorie der Thetafunktionen, insbesondere derer von drei Veränderlichen, Annali di mat., Ser. 2, 9 (1879), 126-166.

\section{The Rockefeller Institute,}

New York 21, N.Y. 